\title{
Conductance spectra of ferromagnetic superconductors: Quantum transport in a ferromagnetic metal/non-unitary ferromagnetic superconductor junction
}

\author{
J. Linder and M. S. Grønsleth \\ Department of Physics, Norwegian University of Science and Technology, N-7491 Trondheim, Norway
}

\author{
A. Sudbø \\ Department of Physics, Norwegian University of Science and Technology, N-7491 Trondheim, Norway and \\ Centre for Advanced Study, Norwegian Academy of Science and papers, Drammensveien 78, N-0271 Oslo, Norway.
}

(Dated: Received September 12, 2018)

\begin{abstract}
Recent findings of superconductors that simultaneously exhibit multiple spontaneously broken symmetries, such as ferromagnetic order or lack of an inversion center and even combinations of such broken symmetries, have led to much theoretical and experimental research. We consider quantum transport in a junction consisting of a ferromagnetic metal and a non-unitary ferromagnetic superconductor. It is shown that the conductance spectra provides detailed information about the superconducting gaps, and is thus helpful in determining the pairing symmetry of the Cooper pairs in ferromagnetic superconductor.
\end{abstract}

PACS numbers: 74.20.Rp, 74.50.+r, 74.20.-z

\section{INTRODUCTION}

Spontaneous symmetry breaking in condensed matter systems ranks among the most profound emergent phenomena in many-body physics. Multiple spontaneously broken symmetries are not only of interest in terms of studying properties of specific condensed matter systems, but also due to the fact that it may provide clues for what could be expected in other systems in vastly different areas of physics. A first attempt $\mathrm{t}^{1}$ at describing the physics of magnetic spin-singlet superconductors was made long ago, and the discovery of ferromagnetic superconductors (FMSCs) $)^{2,3}$ displaying coexisting superconductivity (SC) and ferromagnetism (FM) well below the Curie temperature, has produced a realization of a physically rich system that exhibits simultaneously broken $O(3)$ and $U(1)$ symmetries. Spontaneous breaking of symmetry is responsible for a wide range of physical phenomena, such as the mass differences of elementary particles, phase transitions in condensed-matter systems, and even emergent phenomena in biology ${ }^{4}$. In many cases, the phenomena can in fact be described by the same equations. Thus, a study of ferromagnetic superconductors is of interest not only in terms of having an obvious potential for leading to novel devices in for instance nanotechnology and spintronics, but also from a fundamental physics point of view.

A successful model describing a FMSC demands that two important issues are adressed: $i$ ) the symmetry of the pairing state, and ii) whether the superconducting and ferromagnetic order parameters are coexistent or phase-separated in spacetime. Cooper pairs in conventional superconductors ( $s$-wave) are spin-singlets. Thus, $s$-wave pairing and uniform FM are antagonists 5,6 . Spin-triplet Cooper pairs, however, can carry a net magnetic moment. Such Cooper pairs are presently the prime candidate for explaining the coexistence of FM and SC in e.g. $\mathrm{UGe}_{2}$, and $\mathrm{URhGe}^{2,3}$. In these materials, $\mathrm{SC}$ occurs deep within the ferromagnetic phase. It is therefore natural to view the SC pairing as originating with electrons that also contribute to FM. Thus, the electrons responsible for FM below the Curie temperature $T_{\mathrm{M}}$ condense into Cooper pairs with magnetic moments aligned along the magnetization below the critical temperature $T_{\mathrm{c}}$. While spin-singlet superconductivity coexisting with uniform ferromagnetism appears to be unlikely, it could coexist with helically ordered magnets. Tunneling phenomena in such systems have indeed been considered theoretically ${ }^{7,8}$. This is, however, physically quite different from the case we will study in this paper.

Bulk FMSCs are expected to display an unusual feature, namely the spontaneous formation of an Abrikosov vortex lattice ${ }^{9}$. Uniform superconducting phases have also been investigated $^{10}$, but in a bulk system it seems reasonable to assume that this must be associated with a nonuniform magnetic state ${ }^{7,8}$. A key variable determining whether a vortex lattice appears or not seems to be the strength of the internal magnetization $\mathbf{m}^{11}$. The current experimental data ${ }^{3}$ available for URhGe apparently do not settle this issue unambiguously, while uniform coexistence of FM and SC appears to have been experimentally verified ${ }^{12}$ in $\mathrm{UGe}_{2}$. Moreover, a bulk Meissner state in the FMSC $\mathrm{RuSr}_{2} \mathrm{GdCu}_{2} \mathrm{O}_{8}$ has been reported $^{13}$. No consensus has so far been reached concerning the correct pairing symmetry for the FMSCs, although theoretical considerations strongly suggest that a non-unitary state is favored ${ }^{14,15,16}$. In terms of the $\mathbf{d}_{\mathbf{k}}$-vector formalism (see below), this means that the relation $1\left(\mathbf{d}_{\mathbf{k}} \times \mathbf{d}_{\mathbf{k}}^{*}\right) \neq 0$ is satisfied, which is equivalent to saying that the Cooper pairs carry a net $\operatorname{spin}^{17}$. The study of pairing symmetries in unconventional superconductors has a long tradition ${ }^{18}$ where tunneling currents have played a crucial role. For the case of spin-triplet non-magnetic superconductors, theoretical studies of tunneling to a normal metal or ferromagnet have been suggestive in terms of establishing the correct pairing symmetry for the superconductor ${ }^{19,20}$.

In this paper, we consider quantum transport between two thin films of a non-unitary FMSC and an easy-axis ferromagnet, respectively. We demonstrate how the resulting conductance spectra can be exploited to obtain useful information about the superconducting gaps. The SC and FM order parameters are assumed not to be phase-separated. Moreover, 
the choice of a thin film FMSC is motivated by the fact that the pair-breaking orbital effect on Cooper pairs with an in-plane magnetization is suppressed, and that one will avoid vortices present in the compound if the thickness $t$ of the film is smaller than the diameter of a vortex ${ }^{21}, t<\xi_{0} \ll \lambda_{0}$. Here, $\xi_{0}$ is the coherence length (typical size of Cooper pairs) while $\lambda_{0}$ is the penetration depth (typical radius of vortex). In the cases ${ }^{2.3}$ of $\mathrm{UGe}_{2}$ and URhGe, this amounts to $t \sim 10 \mathrm{~nm}$ which is well within reach of current experimental techniques.

We organize our work as follows. In Sec. II we establish the model and formalism which we will apply to the problem. Results are given in Sec. IIII in addition to a discussion of our findings. Concluding remarks are given in Sec. IV

\section{MODEL AND FORMULATION}

Our model is illustrated in Fig. 1, where the superconducting pairing symmetry is equivalent to that of an $A 2$-phase in ${ }^{3} \mathrm{He}$ [see Ref. 17 and the discussion below Eq. (1)] .

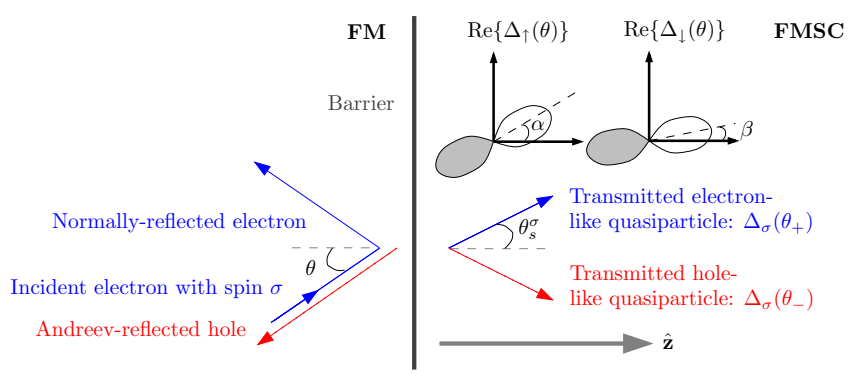

FIG. 1: (Color online). Model system of a FM-FMSC interface in a slab-geometry in the clean limit. Retroreflection symmetry is not broken, since the hole carries the same spin as the incident electron. We have sketched gaps corresponding to the analog ${ }^{17}$ of the A2-phase in liquid ${ }^{3} \mathrm{He}$.

The Bogoliubov-de Gennes (BdG) equations for a FMSC $^{22}$ may be written in compact matrix form

$$
\left(\begin{array}{cc}
\hat{\mathcal{M}}_{\mathbf{k}} & \hat{\Delta}_{\mathbf{k}} \\
\hat{\Delta}_{\mathbf{k}}^{*} & -\hat{\mathcal{M}}_{\mathbf{k}}
\end{array}\right)\left(\begin{array}{c}
u_{\mathbf{k} \sigma} \\
v_{\mathbf{k} \sigma}
\end{array}\right)=E_{\mathbf{k} \sigma}\left(\begin{array}{l}
u_{\mathbf{k} \sigma} \\
v_{\mathbf{k} \sigma}
\end{array}\right)
$$

where we have introduced the $2 \times 2$ matrices

$$
\begin{aligned}
\hat{\mathcal{M}}_{\mathbf{k}} & =\varepsilon_{\mathbf{k}} \hat{1}-\hat{\sigma}_{z} U_{\mathrm{R}}, \\
\hat{\Delta}_{\mathbf{k}} & =\left(\Delta_{0} \hat{1}+\hat{\boldsymbol{\sigma}} \cdot \mathbf{d}_{\mathbf{k}}\right) \hat{\sigma}_{y},
\end{aligned}
$$

in addition to $u_{\mathbf{k} \sigma}=\left[\begin{array}{ll}u_{\mathbf{k} \uparrow \sigma} & u_{\mathbf{k} \downarrow \sigma}\end{array}\right]^{\mathrm{T}}, v_{\mathbf{k} \sigma}=\left[\begin{array}{ll}v_{\mathbf{k} \uparrow \sigma} & v_{\mathbf{k} \downarrow \sigma}\end{array}\right]^{\mathrm{T}}$. Here, $\varepsilon_{\mathbf{k}}$ is a kinetic energy term, $\hat{\boldsymbol{\sigma}}=\left(\hat{\sigma}_{x}, \hat{\sigma}_{y}, \hat{\sigma}_{z}\right)$ is a vector containing the Pauli matrices, $U_{\mathrm{R}(\mathrm{L})}$ describes the magnetic exchange energy of right (left) part of the system. Moreover, the $d_{k}$ vectors are given by

$$
\mathbf{d}_{\mathbf{k}}=\frac{1}{2}\left[\Delta_{\mathbf{k} \downarrow \downarrow}-\Delta_{\mathbf{k} \uparrow \uparrow},-1\left(\Delta_{\mathbf{k} \downarrow \downarrow}+\Delta_{\mathbf{k} \uparrow \uparrow}\right), 2 \Delta_{\mathbf{k} \uparrow \downarrow}\right],
$$

and $\Delta_{0}$ and $\Delta_{\mathbf{k} \alpha \beta}$ are the superconducting spin-singlet and spin-triplet order parameters, respectively. Finally, $u_{\mathbf{k} \sigma}$ and $v_{\mathbf{k} \sigma}$ are the wave-function solutions with eigenvalue $E_{\mathbf{k} \sigma}$. In the following, we set the $\mathrm{k}$-independent singlet amplitude $\Delta_{0}$ to zero, as we do not consider the case of coexistent $s$-wave $\mathrm{SC}$ and $\mathrm{FM}^{16}$. Also, the opposite-spin triplet pairing giving rise to the gap $\Delta_{\mathbf{k} \uparrow \downarrow}$ is in general believed to be suppressed, since it requires interband pairing of Zeeman-split electrons ${ }^{3}$. Hence, our model is that of a non-unitary spin-triplet state with equal-spin pairing, i.e. $\Delta_{\mathbf{k} \uparrow \downarrow}=0, \Delta_{\mathbf{k} \sigma \sigma} \neq 0$, equivalent to the $A 2$-phase in liquid ${ }^{3} \mathrm{He}$ (see e.g. Ref. 17) with a nonvanishing magnetic moment associated with the Cooper pairs.

As indicated in Fig. 1, the reflected and transmitted electron- and hole-like excitations feel different pairing potentials due to the orbital symmetry of the superconducting gaps. The angle into which they are scattered depends on the spin $\sigma$ of the incident electron, since there is a magnetic exchange energy $U_{\mathrm{R}}$ present in the superconducting state. In the following, we will consider the momentum of the quasiparticles as fixed on the Fermi surface for spin $\sigma$ so that the superconducting gaps correspondingly only depend on the direction of momentum, i.e. $\Delta_{\mathbf{k} \sigma \sigma} \rightarrow \Delta_{\sigma}\left(\theta_{s}^{\sigma}\right)$ where $\theta_{s}^{\sigma}$ is defined in Fig. 1. Throughout this paper, we shall insert the superconducting gap a priori instead of solving it self-consistently, in order to obtain analytical formulas. This is justified by the fact that detailed calculations taking into account the modification of the pair potential near the barrier demonstrate that no new qualitative features appear in the conductance spectra compared to when the gap is modelled by a step function at the interface ${ }^{23}$. However, the proximity effect at a FM/SC interface may cause two important phenomena to occur: $i$ ) induction of an SC order parameter (possible mixture of singlet and triplet) in the FM region 24 and ii) the formation of midgap surface states on the interface ${ }^{25}$, leading to a suppression of the OP in the vicinity of the interface. The competition and coexistence of these two phenomena has been studed in Ref. 26. In this work, we will mainly be concerned with a SC pairing symmetry analogous to the A2-phase in liquid ${ }^{3} \mathrm{He}$, for which the latter of these effects is only present for a specific trajectory of the incoming electrons. Thus, we believe our results capture the essential qualitative features of the conductance spectra, although a self-consistent approach would be required in order to obtain the entire picture.

For the simplest model that illustrates the physics, we have chosen a two-dimensional FM-FMSC junction with a barrier modelled by the potential $V(\mathbf{r})=V_{0} \delta(z)$ and superconducting gaps $\Delta_{\sigma}\left(\theta_{s}^{\sigma}, \mathbf{r}\right)=\Delta_{\sigma}\left(\theta_{s}^{\sigma}\right) \Theta(z)$. Here, $\delta(z)$ and $\Theta(z)$ represent the Kronecker delta- and Heaviside functions, respectively. Solving the BdG-equations and applying the BTK formalism ${ }^{27}$, one finds that our system in Fig. 11 is described by the wave-functions for particles and holes with spin $\sigma$ in the ferromagnet $\left(\psi^{\sigma}\right)$ and FMSC $\left(\Psi^{\sigma}\right)$ side of the barrier. Explicitly, the total wave-function $\Psi_{\text {tot }}^{\sigma}(z)=\Theta(-z) \psi^{\sigma}(z)+$ $\Theta(z) \Psi^{\sigma}(z)$ then has the components 


$$
\begin{aligned}
& \psi^{\sigma}(z)=\mathrm{e}^{1 k^{\sigma} \sin \theta y}\left[\left(\begin{array}{l}
1 \\
0
\end{array}\right) \mathrm{e}^{1 k^{\sigma} \cos \theta z}+r_{e}^{\sigma}(E, \theta)\left(\begin{array}{l}
1 \\
0
\end{array}\right) \mathrm{e}^{-1 k^{\sigma} \cos \theta z}+r_{h}^{\sigma}(E, \theta)\left(\begin{array}{c}
0 \\
1
\end{array}\right) \mathrm{e}^{1 k^{\sigma} \cos \theta z}\right], \\
& \Psi^{\sigma}(z)=\mathrm{e}^{1 q^{\sigma} \sin \theta y}\left[t_{e}^{\sigma}(E, \theta)\left(\begin{array}{c}
u_{\sigma}\left(\theta_{s+}^{\sigma}\right) \\
v_{\sigma}\left(\theta_{s+}^{\sigma}\right) \gamma_{\sigma}^{*}\left(\theta_{s+}^{\sigma}\right)
\end{array}\right) \mathrm{e}^{1 q^{\sigma} \cos \theta_{s}^{\sigma} z}+t_{h}^{\sigma}(E, \theta)\left(\begin{array}{c}
v_{\sigma}\left(\theta_{s-}^{\sigma}\right) \gamma_{\sigma}\left(\theta_{s-}^{\sigma}\right) \\
u_{\sigma}\left(\theta_{s-}^{\sigma}\right)
\end{array}\right) \mathrm{e}^{-1 q^{\sigma} \cos \theta_{s}^{\sigma} z}\right],
\end{aligned}
$$

with $\gamma_{\sigma}(\theta)=\Delta_{\sigma}(\theta) /\left|\Delta_{\sigma}(\theta)\right|, \theta_{s+}^{\sigma}=\theta_{s}^{\sigma}, \theta_{s-}^{\sigma}=\pi-\theta_{s}^{\sigma}$. The wave-vectors read $k^{\sigma}=\left[2 m\left(E_{\mathrm{F}}+\sigma U_{\mathrm{L}}\right)\right]^{1 / 2}, q^{\sigma}=$ $\left[2 m\left(E_{\mathrm{F}}+\sigma U_{\mathrm{R}}\right)\right]^{1 / 2}$ while the spin-generalized coherence factors are

$$
u_{\sigma}\left(\theta_{s \pm}^{\sigma}\right)\left[v_{\sigma}\left(\theta_{s \pm}^{\sigma}\right)\right]=\frac{1}{4}\left\{1+[-] \sqrt{1-\left(\left|\Delta_{\sigma}\left(\theta_{s \pm}^{\sigma}\right)\right| / E\right)^{2}}\right\}^{1 / 2} .
$$

In writing down Eq. (4), we have implicitly incorporated conservation of group velocity and conservation of momentum parallell to the barrier, i.e. $k^{\sigma} \sin \theta=q^{\sigma} \sin \theta_{s}^{\sigma}$.

\section{RESULTS AND DISCUSSION}

The normal- and Andreev-reflection coefficients can be calculated by making use of the boundary conditions

$$
\begin{aligned}
& \text { i) } \psi^{\sigma}(0)=\Psi^{\sigma}(0), \\
& \text { ii) } 2 m V_{0} \psi^{\sigma}(0)=\partial /\left.\partial z\left[\Psi^{\sigma}(z)-\psi^{\sigma}(z)\right]\right|_{z=0} .
\end{aligned}
$$

Let us introduce $Z=2 m V_{0} / k_{\mathrm{F}}$ and

$$
\Upsilon_{ \pm}^{\sigma}=q^{\sigma} \cos \theta_{s}^{\sigma} \pm k^{\sigma} \cos \theta \pm 1 k_{F} Z,
$$

while $P_{\sigma}^{\mathrm{L}(\mathrm{R})}=\left(E_{F}+\sigma U_{\mathrm{L}(\mathrm{R})}\right) / 2 E_{F}$ denotes the spin polarization on the left (right) side of the junction. Our calculations then lead to the explicit expressions for the Andreevand normal-reflection coefficients for this FM-FMSC junction, namely

$$
\begin{aligned}
& r_{e}^{\sigma}=-1+2 k^{\sigma} \cos \theta\left[u_{\sigma}\left(\theta_{s+}^{\sigma}\right) u_{\sigma}\left(\theta_{s-}^{\sigma}\right)\left(\Upsilon_{+}^{\sigma}\right)^{*}\right. \\
& \left.\quad+v_{\sigma}\left(\theta_{s-}^{\sigma}\right) v_{\sigma}\left(\theta_{s+}^{\sigma}\right) \gamma_{\sigma}\left(\theta_{s-}^{\sigma}\right) \gamma_{\sigma}^{*}\left(\theta_{s+}^{\sigma}\right)\left(\Upsilon_{-}^{\sigma}\right)^{*}\right] / D^{\sigma} \\
& r_{h}^{\sigma}=4 k^{\sigma} \cos \theta q^{\sigma} \cos \theta_{s}^{\sigma} v_{\sigma}\left(\theta_{s+}^{\sigma}\right) u_{\sigma}\left(\theta_{s-}^{\sigma}\right) \gamma_{\sigma}^{*}\left(\theta_{s+}^{\sigma}\right) / D^{\sigma},
\end{aligned}
$$

upon defining the quantity

$$
\begin{aligned}
D^{\sigma}= & u_{\sigma}\left(\theta_{s+}^{\sigma}\right) u_{\sigma}\left(\theta_{s-}^{\sigma}\right)\left|\Upsilon_{+}^{\sigma}\right|^{2} \\
& -v_{\sigma}\left(\theta_{s-}^{\sigma}\right) v_{\sigma}\left(\theta_{s+}^{\sigma}\right) \gamma_{\sigma}\left(\theta_{s-}^{\sigma}\right) \gamma_{\sigma}^{*}\left(\theta_{s+}^{\sigma}\right) \times\left|\Upsilon_{-}^{\sigma}\right|^{2} .
\end{aligned}
$$

In the limit of weak FM where all quasiparticle momenta equal $k_{\mathrm{F}}$, our results are found to be consistent with a spingeneralized augmentation of the equations in Ref. 29. In their general form, the above equations are novel results that include the effect of magnetism on the superconducting side of the barrier. As demanded by consistency, one obtains total reflection $\left|r_{e}^{\sigma}\right|^{2}=1$ when $\theta>\arcsin \left(q^{\sigma} / k^{\sigma}\right)$. Having obtained the above quantities, one may calculate the conductance $G(E)$ of the setup (in units of the normal conductance, i.e. $\left.\Delta_{\sigma}(\theta) \rightarrow 0\right)$. We find that it is given by

$$
G(E)=\sum_{\sigma} G^{\sigma}(E) / \sum_{\sigma} F^{\sigma}
$$

where we have defined the quantities

$$
\begin{aligned}
G^{\sigma}(E)\left[F^{\sigma}\right] & =\int_{-\pi / 2}^{\pi / 2} \mathrm{~d} \theta \cos \theta G^{\sigma}(E, \theta)\left[f^{\sigma}\right] P_{\sigma}^{\mathrm{L}} P_{\sigma}^{\mathrm{R}}, \\
G^{\sigma}(E, \theta) & =1+\left|r_{h}^{\sigma}(E, \theta)\right|^{2}-\left|r_{e}^{\sigma}(E, \theta)\right|^{2},
\end{aligned}
$$

and $f^{\sigma}=1-\left|1-2 k^{\sigma} \cos \theta / \Upsilon_{+}^{\sigma}\right|^{2}$. We next demonstrate how the conductance spectra yields useful information about the superconducting gaps upon varying the relative orientation of the gaps, their magnitude, and the strength of the magnetic exchange energy on each side of the junction. To be specific, we first first consider the analog of the $A 2$-phase in liquid ${ }^{3} \mathrm{He}$, such that the gaps may be written ${ }^{28}$

$$
\Delta_{\uparrow}\left(\theta_{s \pm}^{\sigma}\right)=-\Delta_{\uparrow, 0} \mathrm{e}^{1\left(\theta_{s \pm}^{\sigma}-\alpha\right)}, \Delta_{\downarrow}\left(\theta_{s \pm}^{\sigma}\right)=\Delta_{\downarrow, 0} \mathrm{e}^{-1\left(\theta_{s \pm}^{\sigma}-\beta\right)},
$$

as illustrated in Fig. 10 We stress that $\theta_{s \pm}^{\sigma}$ is not the global broken $U(1)$ superconducting phase, but rather an internal phase originating with the odd symmetry of the $p$-wave gaps (see Fig. 1). The exchange energy of the FMSC will be kept fixed at $U_{\mathrm{R}}=0.05 E_{\mathrm{F}}$. In Fig. 2, we have plotted the conductance spectra for a FM/FMSC junction for three distinct cases. We have defined the ratio between magnitude of the superconducting gaps as $R_{\Delta}=\Delta_{\uparrow, 0} / \Delta_{\downarrow, 0}$. Introducing the dimensionless barrier strength $Z=2 m V_{0} / k_{\mathrm{F}}$, where $k_{\mathrm{F}}$ is the Fermi momentum, we consider $i$ ) the metallic contact case with no barrier $(Z=0), i i)$ the intermediate case with a moderate barrier $(Z=3)$, and iii) the tunneling limit with an insulator in the junction $(Z \rightarrow \infty)$. For each case $i$ )-iii), we have allowed the magnetic exchange energy $U_{\mathrm{L}}$ to vary from weak, favoring $\uparrow$-spins $\left(U=0.05 E_{\mathrm{F}}\right)$ to strong, favoring $\uparrow$ spins in one case $\left(U=0.5 E_{\mathrm{F}}\right)$ and $\downarrow$-spins in the other case $\left(U=-0.5 E_{\mathrm{F}}\right)$. These are shown in descending order in each column. We have also considered the conductance $G(E)$ for several values of $\{\alpha, \beta\}$. For the gaps chosen above, we find that the $G(E)$ did not depend on different choices of these parameters. This can be understood by observing that the angular averaging over $G^{\sigma}(E, \theta)$ allows for factors $\mathrm{e}^{-1 \alpha(\beta)}$ to be separated out on equal footing as the factor corresponding to the globally broken $U(1)$ symmetry, whose value does not affect the conductance spectra. This will, however, not be the case for other possible triplet symmetries, as discussed below.

An important, and obvious, feature of the conductance spectra is a characteristic behavior occuring at voltages corresponding to $E=\Delta_{\sigma, 0}, \sigma=\uparrow, \downarrow$, where peaks are displayed. This offers the opportunity to utilise the conductance spectra to reap explicit information on the size of the superconducting gaps in the FMSC. From Fig. 2, it is seen that the effect of increasing the exchange energy on the ferromagnetic 
side to $U_{\mathrm{L}}= \pm 0.5 E_{\mathrm{F}}$ is a sharpening of the peaks located at $E=\Delta_{\sigma, 0}$, where $\sigma$ is the spin-species energetically favored by $U_{\mathrm{L}}$. Concomitantly, the peak located at $E=\Delta_{-\sigma, 0}$ is suppressed. Such a response is consistent with what one would expect, since increased/decreased spin polarization on the ferromagnetic side enhances/suppresses the conductance of the corresponding spin component. In the tunneling limit (large
$Z$ ), we see that the conductance spectra exhibits sharp transitions at $E=\Delta_{\sigma, 0}$, corresponding to the sudden appearance of a tunneling current as the voltage exceeds the magnitude of the gaps. We have also considered $G(E)$ in the case of vanishing FM on the left side, i.e. a N/FMSC junction. The results we find are very similar to the case of weak FM displayed in the upper row of Fig. 2, and are therefore not displayed.
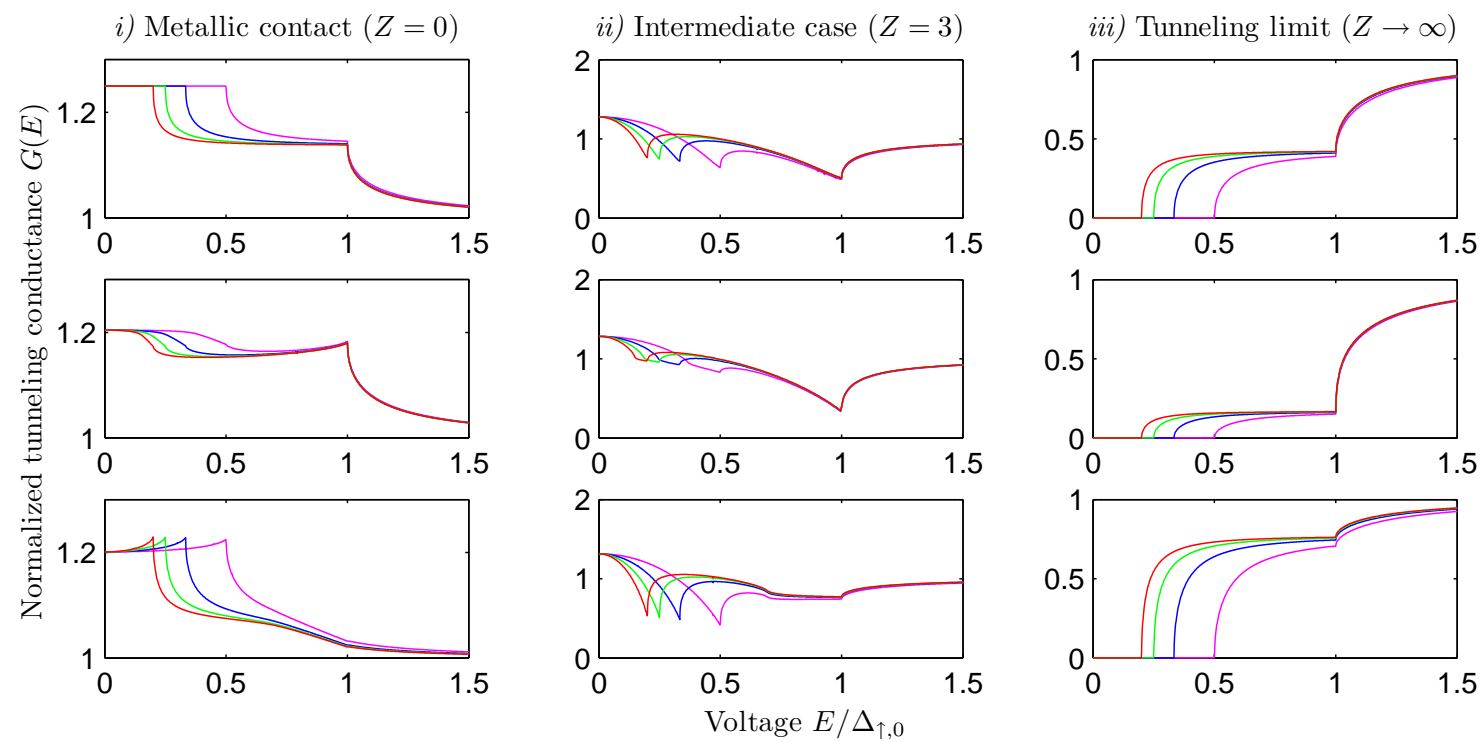

FIG. 2: (Color online). Plot of the conductance $G(E)$ for a FM/FMSC junction in the case of a metallic contact, the tunneling limit, and an intermediate case. The gaps are given by Eq. (12) in this case, for which $G(E)$ is found to be insensitive to $\{\alpha, \beta\}$. In the above, $\{\alpha, \beta\}=0$. The columns for each case provide the spectrum for $U_{\mathrm{L}}=0.05 E_{\mathrm{F}}, U_{\mathrm{L}}=0.5 E_{\mathrm{F}}$, and $U_{\mathrm{L}}=-0.5 E_{\mathrm{F}}$, in descending order. For each figure, we have plotted several ratios between the magnitude of the superconducting gaps. These are given by $R_{\Delta}=\{2,3,4,5\}$, represented by the magenta, blue, green, and red line, respectively.

For the gaps in Eq. (12), the conductance was found to be insensitive to $\{\alpha, \beta\}$. However, in general this is different, and the orientation of the gaps relative to the barrier is essential in determining the conductance spectrum. We illustrate this with a somewhat different choice of anisotropic gaps. When the superconducting gap is oriented in a fashion that leaves it invariant under inversion of the component of momentum perpendicular to the junction, $k_{z} \rightarrow-k_{z}$ in this case (equivalently $\theta \rightarrow \pi-\theta$ ), no zero-bias conductance peak (ZBCP) should be expected as there is no formation of current-carrying zero energy states ${ }^{25}$. However, when the gap changes sign under such an inversion of momentum, ZBCPs $\operatorname{appear}^{29}$. Since the momentum perpendicular to the junction of the hole-like excitation in the anisotropic superconductor is reversed compared to the electron-like excitation, a gap that satisfies $\Delta_{\sigma}(\theta) \neq \Delta_{\sigma}(\pi-\theta)$ will cause the hole to feel a different pairing potential than the electron-like excitation. This is the fundamental reason for the appearance of a ZBCP. However, in the present case of $p$-wave superconducting gaps analogous to the $A 2$-phase in ${ }^{3} \mathrm{He}$, both gaps are asymmetric un- der the operation $\theta \rightarrow \pi-\theta$ regardless of the value of $\{\alpha, \beta\}$ except for the single value $\theta=0$. Therefore, a small contribution to zero energy current-carrying states, i.e. $G(0) \neq 0$, will occur as shown in Fig. 2. However, this contribution does not lead to a ZBCP, for which all $\theta$ (see Fig. 2) contribute to the formation of zero energy states due to the asymmetry of the gaps. In a model where the $p$-wave gaps are represented by the odd-symmetry analog of $d_{x^{2}-y^{2}}$-wave gaps, i.e.

$$
\Delta_{\uparrow}(\theta)=\Delta_{\uparrow, 0} \cos (\theta-\alpha), \Delta_{\downarrow}(\theta)=\Delta_{\downarrow, 0} \cos (\theta-\beta),
$$

the formation of $\mathrm{ZBCP}$ will then be predictable from the orientation of the gaps as these can now display symmetry/antisymmetry/asymmetry when $\theta \rightarrow \pi-\theta$, depending on $\{\alpha, \beta\}$ (see Fig. 1). Indeed, insertion of the above gaps into our model produces conductance spectra that display a ZBCP for e.g. $\alpha=\beta=0$, as can be seen in Fig. 3. In this case, as in Fig. 2, the conductance spectra also allows for the magnitude of the superconducting gaps to be read out, although the features are not as clear as those seen in Fig. 2 

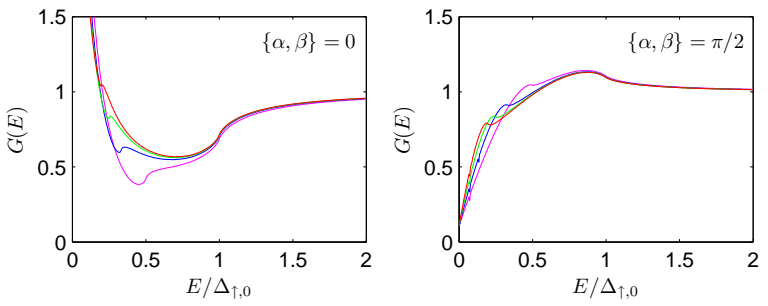

FIG. 3: (Color online). Plot of the conductance $G(E)$ for FM/FMSC junction with gaps given by Eq. [13, for which $G(E)$ is sensitive to $\{\alpha, \beta\}$. We have chosen $Z=3$, and $R_{\Delta}=\{2,3,4,5\}$ are represented by the magenta, blue, green, and red line, respectively.

From the results of Figs. 2 and 3 , it is clear that the conductance spectra $G(E)$ exhibit strong dependence on the exchange energy, while the relative orientation of the gaps is insignificant for the superconducting gaps Eq. (12). Thus, our results will shed light on the magnitude of the various components of the superconducting gaps and their relative orientations in $\mathbf{k}$-space if the gaps display symmetry/antisymmetry under $\theta \rightarrow \pi-\theta$ for some orientation. Moreover, we are dealing with an easily observable effect, since distinguishing between the peaks occuring for various values of $R_{\Delta}$ requires a resolution of order $\mathcal{O}\left(10^{-1} \Delta_{\uparrow, 0}\right)$, which typically corresponds to $0.1-1 \mathrm{meV}$. These structures should be then readily resolved with present-day STM technology. However, in order to do justice to the experimentalist, it should be pointed out that a challenge with respect to tunneling junctions is dealing with non-idealities at the interface which may affect the conductance spectrum. Also, the importance of spin-flip processes in the vicinity of the interface (if such are indeed present) has recently been pointed out ${ }^{30}$.

\section{CONCLUSION}

In summary, we have studied the conductance spectra $G(E)$ for systems consisting of a ferromagnetic metal separated from a non-unitary $p$-wave FMSC by a thin, insulating barrier. We have considered the cases of weak, intermediate, and strong barriers, and considered three different strengths of the ferromagnetic exchange energy. We have considered two classes of anisotropic spin-triplet superconducting gaps, with results given in Figs. (2) and (3). Our results show how the magnitude of the superconducting gaps $\Delta_{\sigma}, \sigma=\uparrow, \downarrow$ may be inferred from the conductance spectra. Moreover, the class of superconducting gaps given in Eq. (12) renders $G(E)$ insensitive to $\{\alpha, \beta\}$, the results are shown in Fig. (2) for $\{\alpha, \beta\}=0$. Conversely, the orientations of the $p$-wave gaps modelled by Eq. (13), specific values of $\{\alpha, \beta\}$ are essential to the formation of ZBCPs in $G(E)$ in Fig. (3), and the characteristic behavior at $E=\Delta_{\sigma, 0}, \sigma=\uparrow, \downarrow$. These results should provide useful insights in determining both the relative orientation between the superconducting gaps, as well as their magnitude, in ferromagnetic spin-triplet superconductors.

\section{Acknowledgments}

This work was supported by the Norwegian Research Council Grants No. $157798 / 432$ and No. 158547/431 (NANOMAT), and Grant No. 167498/V30 (STORFORSK).
1 V. L. Ginzburg, Zh. Eksp. Theor. Fiz. 31, 202 (1956).

2 S. S. Saxena et al., Nature 406, 587 (2000).

${ }^{3}$ D. Aoki et al., Nature 413, 613 (2001).

${ }^{4}$ P. W. Anderson, Basic Notions of Condensed Matter Physics, Addison Wesley (1980).

5 E. I. Blount and C. M. Varma, Phys. Rev. Lett., 42, 1079 (1979); E. I. Blount and C. M. Varma, Phys. Rev. Lett. 43, 1843 (1979).

${ }^{6}$ R. Shen, Z. M. Zheng, S. Liu, D. Y. Xing, Phys. Rev. B 67, 024514 (2003).

7 M. L. Kulic and I. M. Kulic, Phys. Rev. B 63, 104503 (2001); M. L. Kulic, C. R. Physique, 7, 4 (2006); cond-mat / 0508276.

${ }^{8}$ I. Eremin, F. S. Nogueira, and R.-J. Tarento, Phys. Rev. B 73, 054507 (2006).

9 S. Tewari, D. Belitz, T. R. Kirkpatrick, J. Toner, Phys. Rev. Lett. 93, 177002 (2004).

10 D. V. Shopova, D. I. Uzunov, Phys. Rev. B 72, 024531 (2005).

11 V. P. Mineev, K. V. Samokhin, Introduction to Unconventional Superconductivity, Gordon and Breach, New York (1999).

${ }_{12}$ H. Kotegawa et al., J. Phys. Soc. Jpn. 74, 705 (2005).

${ }^{13}$ C. Bernhard, J. L. Tallon, E. Brücher, R. K. Kremer, Phys. Rev. B 61, R14960 (2000).

${ }^{14}$ F. Hardy, A. D. Huxley, Phys. Rev. Lett. 94, 247006 (2005).

15 K. V. Samokhin, M. B. Walker, Phys. Rev. B 66, 174501 (2002).

${ }^{16}$ K. Machida, T. Ohmi, Phys. Rev. Lett. 86, 850 (2001).
17 A. J. Leggett, Rev. Mod. Phys. 47, 331 (1975).

18 T. Yokoyama, Y. Tanaka, and J. Inoue, Phys. Rev. B 72, 220504(R) (2005); R. Kleiner et al., Phys. Rev. Lett. 76, 2161 (1996); J. H. Xu, J. L. Shen, J. H. Miller, Jr., and C. S. Ting, Phys. Rev. Lett. 73, 2492 (1994).

19 C. J. Bolech and T. Giamarchi, Phys. Rev. Lett. 92, 127001 (2004).

20 N. Stefanakis, Phys. Rev. B 65, 064533 (2002); N. Stefanakis, Phys. Rev. B 64, 224502 (2001).

21 R. Meservey, P. M. Tedrow, Phys. Rep. 238, 173 (1994).

22 B. J. Powell, J. F. Annett, B. L. Györffy, J. Phys. A: Math. Gen. 36, 9289 (2003).

23 Y. S. Barash, H. Burkhardt, D. Rainer, Phys. Rev. Lett. 77, 4070 (1996); Y. Tanaka and S. Kashiwaya, Phys. Rev. B 58, 2948 (1998); Y. Tanaka, T. Asai, N. Yoshida, J. Inoue, S. Kashiwaya, Phys. Rev. B 61, R11902 (2000).

${ }^{24}$ M. Eschrig, J. Kopu, J. C. Cuevas, G. Schon, Phys. Rev. Lett. 90, 137003 (2003).

25 C.-R. Hu, Phys. Rev. Lett. 72, 1526 (1995).

26 Y. Tanaka, Y. V. Nazarov and S. Kashiwaya, Phys. Rev. Lett. 90, 167003 (2003).

27 G. E. Blonder, M. Tinkham, and T. M. Klapwijk, Phys. Rev. B 25, 4515 (1982).

28 A. P. Mackenzie and Y. Maeno, Rev. Mod. Phys. 75, 657 (2003). 
${ }^{29}$ Y. Tanaka and S. Kashiwaya, Phys. Rev. Lett. 74, 3451 (1995).

${ }^{30}$ S. Kreuzer et al., Appl. Phys. Lett. 80, 4582 (2002). 